\section{Deleterious systemic effects of OSA: how much evidence do we need?}

\author{
Malcolm Kohler ${ }^{1,2,3}$
}

There is accumulating evidence from welldesigned observational cohort studies and randomised controlled trials suggesting that obstructive sleep apnoea syndrome (OSAS) may independently add to vascular risk and increased mortality. Major biological mechanisms underpinning the association between OSAS and systemic consequences such as cardiovascular disease are thought to include intermittent hypoxia leading to increased oxidative stress and sympathetic activity, intrathoracic pressure changes leading to excessive mechanical stress on the heart and large artery walls and arousal-induced reflex sympathetic activation with resultant repetitive large blood pressure rises. ${ }^{1}$ The nocturnal sympathetic nervous system activation and consequent elevation of blood pressure diminish its usual physiological nocturnal dipping. Augmented sympathetic activation in patients with OSAS has also been shown to be associated with blunted baroreflex sensitivity, increased arterial stiffness and impaired endothelial function, all of which contribute to the development of arterial hypertension and consecutive vascular disease. $^{23}$

Treatment with CPAP and mandibular advancement devices (MAD) have been shown to effectively abolish apnoeas and oxygen desaturations, and to prevent arousals; thus, obviate acute blood pressure swings. More than 40 randomised controlled trials looking at the effect of CPAP or MAD therapy on blood pressure have been conducted in the past and the findings of these trials have established that CPAP treatment of patients with OSAS lowers blood pressure between 2 and $10 \mathrm{~mm} \mathrm{Hg}$ after several weeks of CPAP therapy. ${ }^{245}$

What do the three papers published in the current issue of the journal add to the literature? ${ }^{6-8}$ Two of the three papers ${ }^{6} 7$ stem from the Multi-Ethnic Study of Atherosclerosis (MESA), a multisite cohort

\footnotetext{
${ }^{1}$ Division of Pulmonology, University Hospital of Zurich, Zurich, Switzerland; ${ }^{2}$ Center for Integrative Human Physiology, University of Zurich, Zurich, Switzerland; ${ }^{3}$ Center for Interdisciplinary Sleep Research, University of Zurich, Zurich, Switzerland

Correspondence to Prof Malcolm Kohler, Division of Pulmonology, University Hospital of Zurich, Raemistrasse 100, Zurich 8091, Switzerland; Malcolm.Kohler@usz.ch
}

study of community-dwelling men and women aged $45-84$ years without known cardiovascular disease at enrolment. ${ }^{9}$ A subset of these patients participated in the MESA sleep ancillary study from which the presented data were drawn. In the study by Lutsey et $a l,^{6}$ the hypothesis was tested whether polysomnography-derived indices of OSA severity and sleep quality are associated with the prevalence of coronary artery calcification (CAC) in coronary CT. The authors found that patients with severe OSA were more likely to have prevalent CAC relative to those with no evidence of OSA $(1.10,95 \%$ CI 1.01 to 1.19$)$. A high burden of CAC was associated with sleep fragmentation (arousal index) and impaired sleep quality (shorter time in stage N3, slow wave sleep (SWS)). These findings make sense from a physiological point of view as both of these measures of sleep quality (increased arousal index, diminished SWS) are associated with increased levels of sympathetic nervous system activity and hypertension which may be the underlying mechanisms for the observed association between OSA and CAC. ${ }^{10}$

In the study by Kwon et $a l^{7}$ the crosssectional association between atrial fibrillation (AF) and polysomnography-derived measures of sleep breathing disturbances and sleep quality was investigated. There was a weak association between higher apnoea/hypopnoea index and the odds for AF after adjustment for covariates (OR: 1.22, 95\% CI 0.99 to 1.49). However, there was a significantly lower odds for AF with longer duration of SWS (OR: 0.68, 95\% CI 0.51 to 0.92 ) even after adjustment for covariates. Thus, in both abovementioned studies there was some association between OSA severity and the outcomes, but the interesting finding was really that there seems to be a relevant association between impaired sleep quality and an adverse outcome (CAC and AF). This raises the hypothesis that poor sleep quality (ie, reduced SWS) itself may be causally involved in the pathogenesis of subclinical coronary atherosclerosis and AF, possibly through the activation of the sympathetic nervous system. Such an association between impaired sleep quality and subclinical measures of cardiovascular disease has recently been described in other sleep disorders apart from OSAS (eg, in patients with insomnia) ${ }^{11}$ and this observation warrants further well-designed interventional trials to establish the proposed causal relationship.

The third paper by Molnar et $a l^{8}$ published in this issue of the journal stems from the large Racial and Cardiovascular Risk Anomalies in Chronic Kidney Disease (RCAV) study, which examines risk factors for incident chronic kidney disease (CKD) in more than 3 million US veterans with a baseline glomerular filtration rate (GFR) $\geq 60 \mathrm{~mL} / \mathrm{min} / 1.73 \mathrm{~m}^{2} .^{12}$ The authors investigated the possible association between incident OSA, either treated with CPAP or untreated, and (1) all-cause mortality, (2) incident coronary heart disease (CHD), (3) incident strokes, (4) incident CKD (estimated GFR (eGFR) $<60 \mathrm{~mL} / \mathrm{min} /$ $1.73 \mathrm{~m}^{2}$ ) and (5) slopes of eGFR.

Patients with untreated and treated OSA had an $86 \%$ (HR 1.86, 95\% CI 1.81 to 1.91) and $35 \%$ (HR 1.35, 95\% CI 1.21 to 1.51) higher mortality risk relative to patients without OSA. Similarly, untreated as well as treated OSA was associated with a substantially higher risk of incident CHD, strokes and CKD. In addition, the slope of eGFR was more than doubled in untreated patients with OSA $(-0.87$ (IQR -3.00 to 0.70$\left.) \mathrm{mL} / \mathrm{min} / 1.73 \mathrm{~m}^{2}\right) \mathrm{com}$ pared with patients without OSA $(-0.41$ (IQR -2.01 to -0.99 ) $\mathrm{mL} / \mathrm{min} / 1.73 \mathrm{~m}^{2}$ ). This is in line with previous reports suggesting that OSA may promote progression of kidney disease and increase filtration fraction. ${ }^{13-15}$ As the diagnosis of OSA and treatment with CPAP in the study by Molnar et $a l^{8}$ were drawn from ICD-9-CM (The International Classification of Diseases, Ninth Revision, Clinical Modification) codes, there are no data available on the actual sleep study and thus the severity of OSA. In addition, the real impact of CPAP treatment on kidney function is difficult to assess because there are no data on actual CPAP usage. Last but not least, potential underlying mechanisms remain speculative. Again increased sympathetic activity, increased blood pressure and consecutive endothelial dysfunction seem likely mechanisms to explain the observed association between OSA and impaired renal function.

Although such large observational studies have added significantly to the literature in the past and still do, they are prone to bias and are unable to establish a causal relationship between OSAS and its proposed detrimental systemic consequences. To date there are no data from randomised controlled trials, proving that treatment of OSAS is associated with fewer 
cardiovascular events and improved mortality. Furthermore, observational studies do not allow a thorough investigation of the underpinning biological mechanisms, which seems crucial if we want to understand the disease and advance the development of novel treatment strategies. So is there enough evidence to inform our colleagues from cardiology, neurology and nephrology that we have found a modifiable risk factor and they should look out for it? I doubt it. This would require yet another approach to answer the question. A real-life screening trial looking at the long-term effect of randomising high-risk subjects for OSA to either a sleep study or none would be a possibility. If such a screening trial would show that relevant outcomes, that is, cardiovascular events and mortality, can be improved by performing a sleep study and initiating treatment if indicated, then we would indeed have enough evidence. To have enough power such a screening trial would need to include tens of thousands of participants to detect a clinically relevant difference in vascular event rates. So the books are not closed yet, let's get working!

Competing interests None declared.

Provenance and peer review Commissioned; internally peer reviewed.

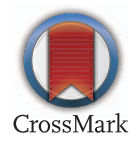

To cite Kohler M. Thorax 2015;70:817-818.

Published Online First 14 July 2015

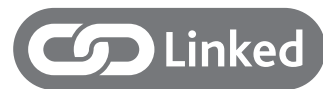

- http://dx.doi.org/10.1136/thoraxjnl-2015-206871

- http://dx.doi.org/10.1136/thoraxjnl-2014-206655

- http://dx.doi.org/10.1136/thoraxjnl-2015-206970

Thorax 2015;70:817-818.

doi:10.1136/thoraxjn-2015-207247

\section{REFERENCES}

1 Kohler M, Stradling JR. Mechanisms of vascular damage in obstructive sleep apnea. Nat Rev Cardiol 2010;7:677-85.

2 Kohler M, Pepperell JC, Casadei B, et al. CPAP and measures of cardiovascular risk in males with OSAS. Eur Respir J 2008;32:1488-96.

3 Kohler M, Stoewhas AC, Ayers L, et al. Effects of continuous positive airway pressure therapy withdrawal in patients with obstructive sleep apnea: a randomized controlled trial. Am J Respir Crit Care Med 2011;184:1192-9.

4 Becker HF, Jerrentrup A, Ploch T, et al. Effect of nasal continuous positive airway pressure treatment on blood pressure in patients with obstructive sleep apnea. Circulation 2003;107:68-73.

5 Haentjens P, Van Meerhaeghe A, Moscariello A, et al. The impact of continuous positive airway pressure on blood pressure in patients with obstructive sleep apnea syndrome: evidence from a meta-analysis of placebo-controlled randomized trials. Arch Int Med 2007;167:757-64.

6 Lutsey PL, McClelland RL, Duprez D, et al. Objectively measured sleep characteristics and prevalence of coronary artery calcification: the Multi-
Ethnic Study of Atherosclerosis Sleep study. Thorax 2015;70:880-7.

7 Kwon Y, Gharib SA, Biggs ML, et al. Association of sleep characteristics with atrial fibrillation: the MultiEthnic Study of Atherosclerosis. Thorax 2015;70:873-9.

8 Molnar MZ, Mucsi I, Novak M, et al. Association of incident obstructive sleep apnoea with outcomes in a large cohort of US veterans. Thorax 2015;70:888-95.

9 Bild DE, Bluemke DA, Burke GL, et al. Multi-ethnic study of atherosclerosis: objectives and design. Am J Epidemiol 2002;156:871-81.

10 Fung MM, Peters K, Redline $S$, et al. Decreased slow wave sleep increases risk of developing hypertension in elderly men. Hypertension 2011; 58:596-603.

11 Carroll JE, Seeman TE, Olmstead R, et al. Improved sleep quality in older adults with insomnia reduces biomarkers of disease risk: pilot results from a randomized controlled comparative efficacy trial. Psychoneuroendocrinology 2015;55:184-92.

12 Gosmanova EO, Lu JL, Streja E, et al. Association of medical treatment nonadherence with all-cause mortality in newly treated hypertensive US veterans. Hypertension 2014;64:951-7.

13 Szentkiralyi A, Czira ME, Molnar MZ, et al. High risk of obstructive sleep apnea is a risk factor of death censored graft loss in kidney transplant recipients: an observational cohort study. Sleep Med 2011;12: 267-73.

14 Stehouwer CD, Gall MA, Twisk JW, et al. Increased urinary albumin excretion, endothelial dysfunction, and chronic low-grade inflammation in type 2 diabetes: progressive, interrelated, and independently associated with risk of death. Diabetes 2002;51: 1157-65.

15 Kinebuchi S, Kazama JJ, Satoh M, et al. Short-term use of continuous positive airway pressure ameliorates glomerular hyperfiltration in patients with obstructive sleep apnoea syndrome. Clin Sci 2004; 107:317-22. 